\title{
Observations of sensor bias dependent cluster centroid shifts in a prototype sensor for the $\mathrm{LHCb}$ Vertex Locator detector*
}

\author{
A. Papadelis on behalf of the LHCb VELO group \\ National Institute for Nuclear Physics and High Energy Physics (NIKHEF) \\ P.O. Box 41882, 1009 DB Amsterdam, The Netherlands \\ Email: arasp@nikhef.nl
}

\begin{abstract}
We present results from a recent beam test of a prototype sensor for the LHCb Vertex Locator detector, read out with the Beetle1.3 front-end chip. We have studied the effect of the sensor bias voltage on the reconstructed cluster positions in a sensor placed in a $120 \mathrm{GeV}$ pion beam at a $10^{\circ}$ incidence angle. We find an unexplained systematic shift in the reconstructed cluster centroid when increasing the bias voltage on an already overdepleted sensor. The shift is independent of strip pitch and sensor thickness.
\end{abstract}

*To be published in the proceedings of the Vertex 2005 workshop, Lake Chuzenji, Nikko, Japan, November 7 - 11 2005. Preprint submitted 22 December 2005. 


\section{Introduction}

The Vertex Locator (VELO) is one of the silicon tracking detectors in the LHCb detector, designed for operation at the Large Hadron Collider (LHC) at CERN. The LHCb detector is a one arm spectrometer designed to measure $\mathrm{CP}$-violation in the B-system and detect rare B-decays. The VELO consists of 84 half circle shaped n-on-n micro strip sensors with $\mathrm{R}$ and $\phi$ geometry. The active region starts at $8.2 \mathrm{~mm}$ from the beam and extends to $42 \mathrm{~mm}$. The strip pitch of the R-sensors increases linearly with the radius, from 40 to $101 \mu \mathrm{m}$. The VELO and LHCb experiment are described in detail in Refs. [1] and [2].

The main purpose of the VELO is the reconstruction of primary and secondary vertices. For this, excellent experimental accuracy is needed, which can only be achieved with an accurate alignment of the VELO sensors. In this paper we present results from beam tests of 2 unirradiated VELO sensors. We observe problems in the sensor alignment that may show up during $\mathrm{LHCb}$ running, unless the origin of the problems is understood.

\section{Experimental setup}

Two unirradiated R-measuring prototype sensors of $200 \mu \mathrm{m}$ and $300 \mu \mathrm{m}$ thickness (hereafter referred to as R200 and R300) were read out with Beetle1.3 front-end (FE) chips [3] set to a peaking time of about $25 \mathrm{~ns}$. The depletion voltage of the $200 \mu \mathrm{m}(300 \mu \mathrm{m})$ sensor is measured to be $34 \mathrm{~V}(55 \mathrm{~V})$. At $100 \mathrm{~V}$ bias the average drift time of the electrons is expected to be $4 \mathrm{~ns}$ ( $8 \mathrm{~ns}$ ), which is well below the peaking time of the FE chip. The sensors were placed in the SPS $120 \mathrm{GeV}$ pion beam in the $\mathrm{X} 7$ area at CERN. One quarter of a sensor was read out, covering the full pitch range. A separate beam telescope [4] provided the tracks. The use of a scintillator trigger asynchronous to the Beetle sampling clock enables full pulse shape determination. Each event was stamped with a TDC time value, which can be used offline to select events that were sampled at their peak signal value.

The normals to the sensors were positioned at a $10^{\circ}$ angle of incidence relative to the beam. Data were recorded at $100 \mathrm{~V}, 200 \mathrm{~V}$ and $300 \mathrm{~V}$ sensor bias, consecutively and without applying any other change to the experimental setup.

\section{Analysis}

The analysis is performed within the framework of VeloTB [5], a software package developed for VELO beam tests. It provides tools for track reconstruction, alignment, clustering and histogram generation. The histograms are saved in a Root file [6].

The track algorithms are described in detail in Ref. [7]. Clusters are seeded by strips that pass a Signal to Noise $(\mathrm{S} / \mathrm{N})$ threshold of 6 . Neighbouring strips passing a threshold of $10 \%$ of the seeding strip $\mathrm{S} / \mathrm{N}$ value are added to the cluster. The cluster size is limited to 5 strips but at $10^{\circ}$ beam incidence angle the vast majority of the clusters are 1 or 2 strip clusters. The cluster position is calculated using the weighted mean of the strip positions with respect to their charge, taking advantage of charge sharing in the sensor to increase the position resolution. The track residual is defined as the distance between a cluster centre and the track intercept point on the sensor as determined by the telescope. We use the standard deviation of a Gaussian fit to the residuals distribution as a measure 
of the spatial resolution of our detector.

The alignment is done by minimising a $\chi^{2}$ built from the residuals between track intercept points and clusters. The $\chi^{2}$ is minimised using an iterative technique implemented using Minuit [8]. The positions of the telescope and the sensors were kept unchanged during the entire data taking period. We therefore perform the alignment using the $100 \mathrm{~V}$ data and use the outcome in the analysis of the 200 and $300 \mathrm{~V}$ data. Due to alignment problems, the strips in the fine pitch region $(40-50 \mu \mathrm{m})$ are not included in the analysis.

The full datasets contain about 310 000, 410000 and 470000 events at 100, 200 and $300 \mathrm{~V}$, respectively. To minimise the presence of noise clusters in the analysis only events passing a \pm 5 ns TDC-cut around the peak of the pulse shape ${ }^{1}$ are included. Clusters with or adjacent to dead or noisy strips are excluded. Only events with tracks that point to a fiducial region of the sensors are included. To correct for cross talk in the setup a digital signal filter ${ }^{2}$ is applied to the data offline.

\section{Results}

A Gaussian function is fitted to the track residuals distribution and the fitted mean value is plotted versus the radius of the strips. Figures $1 \mathrm{a}$ and $\mathrm{b}$ show this for the different bias voltages and two sensor thicknesses. The standard deviation of the residuals distribution ranges from $10-25 \mu \mathrm{m}$, depending on strip pitch region. The fitted mean of a perfectly aligned sensor ${ }^{3}$ should be 0 . The crucial observation is that the 200 and $300 \mathrm{~V}$ points show a shift towards negative values. The shift looks constant in the entire radial range ${ }^{4}$ of the sensor. The residuals mean shift relative to the $100 \mathrm{~V}$ data is shown in Figure 2 . The average shift at $200 \mathrm{~V}$ bias for a R200 (R300) sensor is 3.6 $\pm 0.1(3.6 \pm 0.1) \mu \mathrm{m}$, and at $300 \mathrm{~V} 8.1 \pm 0.1(8.9 \pm 0.1)$. From first order polynomial fits to the data points of Figure 2 no significant pitch dependence can be deduced. Of interest is also the observation that the standard deviation of the residual distributions does not change between the sensor bias settings.

Based on the absence of any time dependence of the residuals mean within each dataset, we can exclude the possibility that slow sensor displacement is the source of the apparent shift. We therefore believe that the shift is directly related to the change in sensor bias. The possibility of sensor displacements due to electrostatic forces related to the sensor bias can not be excluded but a more plausible explanation is that the shift is due to a change of conditions in the silicon.

By measuring the charge on the two strips surrounding the track intercept point we can calculate the quantity $\eta=Q_{\text {inner }} /\left(Q_{\text {inner }}+Q_{\text {outer }}\right)$, where $Q_{\text {inner }}\left(Q_{\text {outer }}\right)$ is the charge in the strip at smaller (larger) radius with respect to the track. We let $\eta(x)$ represent the mean $\eta$ value of all tracks intercepting the sensor at interstrip position $x(0 \leq x \leq 1)$. One expects $\eta(0.5)=0.5$ for a well aligned detector, because a particle that traverses the detector exactly between two strips is expected to generate equally big signals in both strips. In the case of a misaligned sensor, or if the particle for any reason does not induce

\footnotetext{
${ }^{1}$ The pulse peaking time is about 25 ns.

${ }^{2}$ The filter is a 5 th order Finite Impulse Response filter, the coefficients of which are based on beam measurements.

3 The reasons behind the "banana" shape of the R200 sensor are not yet fully understood. One hypothesis is that it is due to a warped sensor.

${ }^{4}$ We remind the reader that the strip pitch increases with the radius.
} 

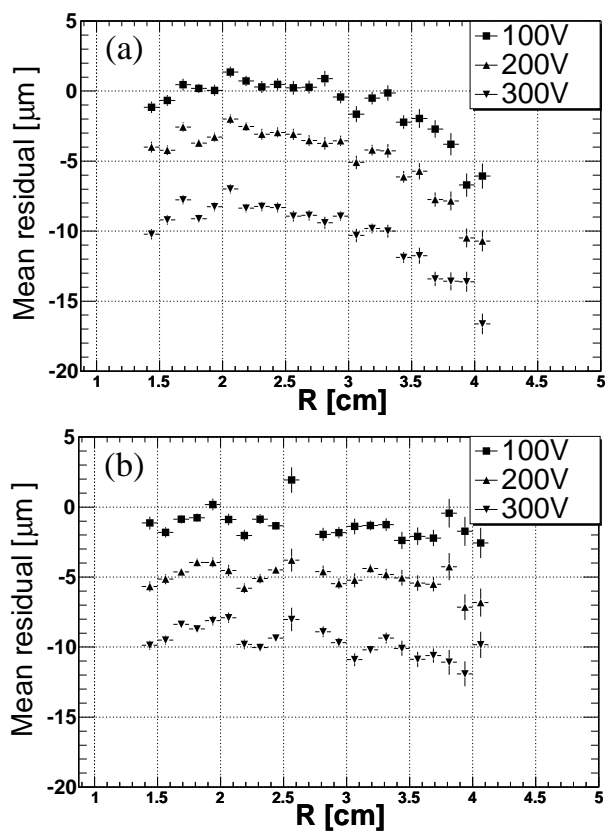

Figure 1: The residuals mean versus strip radius for the R200 (a) and R300 (b) sensors. The error bars represent the statistical error on the fitted mean. The lack of the data points around $2.7 \mathrm{~cm}$ radius in (b) is due to a dead read out chip.

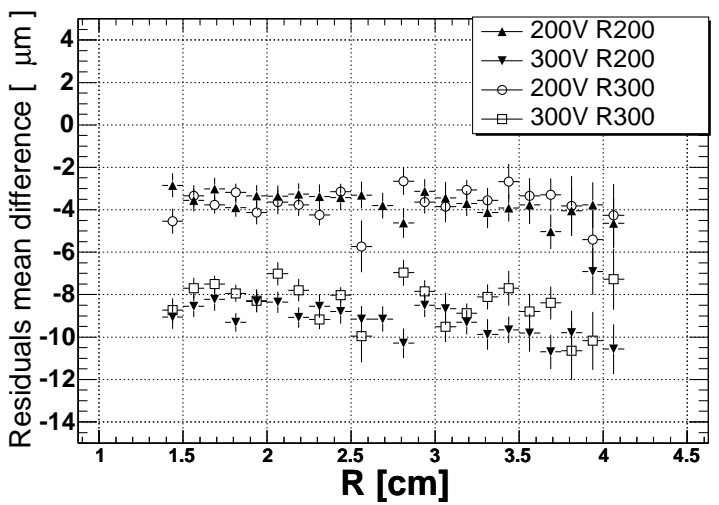

Figure 2: The residuals mean shift relative to the $100 \mathrm{~V}$ data for both thicknesses.

equal amounts of charge in the two strips, $\eta(0.5) \neq 0.5$. Figure 3 shows the situation for the R300 sensor. Only the $100 \mathrm{~V}$ data is symmetric around $x=0.5$.

One of the possible explanations for this effect is ballistic deficit. It occurs if the electron drift times can not be considered small compared to the Beetle shaping time of $25 \mathrm{~ns}$. Since the drift velocity is proportional to the E-field in the sensor, the $100 \mathrm{~V}$ data is most likely to suffer from ballistic deficit. With large drift times, the electrons close to the p-side will not contribute fully to the generated strip signal. This can cause a shift in the cluster centroid relative to the case of less ballistic deficit and would consequently also show up in the $\eta$ distribution in Fig. 3. The direction of the observed shift agrees with this hypothesis. A pictorial representation of ballistic deficit is shown in Figure 4.

Since we operate the sensors well above their depletion voltage, the effects of ballistic deficit are likely to show up as a sensor thickness dependence of the residuals shift. However, our measurements show that the shift is very weakly depending on the thickness (see 


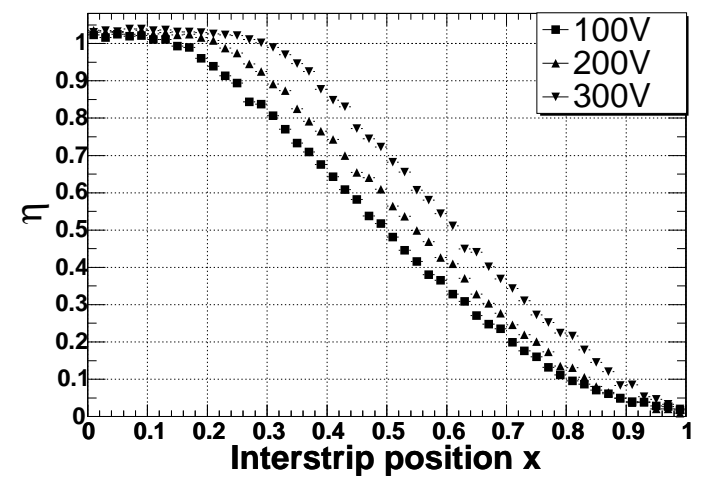

Figure 3: The fitted mean of the $\eta$ distribution versus the interstrip position $x$ of the track intercept point for the R300 sensor.
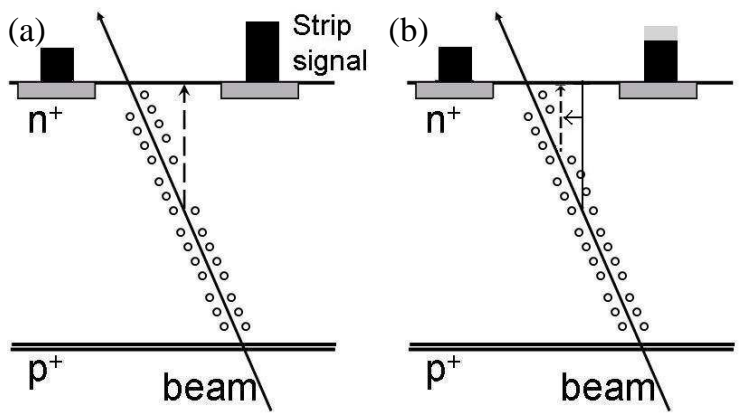

Figure 4: The effect of ballistic deficit for an angled track. The picture is not drawn to scale. The left (right) strip corresponds to the inner (outer) strip in the definition of $\eta$ in Sec. 4. The dashed arrow marks the cluster centroid. In (a) all the charge is collected on the strips in time. In (b) the electrons close to the p-side do not reach the strips in time to contribute fully. Hence, the signal on the right strips decreases (marked by a smaller signal box on the right strip in the figure), which changes the charge distribution between the strips and causes the measured cluster centre to shift towards the left.

Figure 2). Although not fully conclusive, it is a strong argument against this hypothesis. Another argument against ballistic deficit being a major contributor to the shift is that the collected charge at $100 \mathrm{~V}$ is within $90 \%$ of the collected charge at $300 \mathrm{~V}$ for both sensor thicknesses.

\section{Conclusions and outlook}

We have observed a shift in the mean value of the track residuals distribution when increasing the sensor bias on an already overdepleted sensor. The shift shows very weak, if any, dependence on the sensor thickness. A study of the eta(x) function shows that the point where equal charge sharing between the strips occurs is displaced from the interstrip position $x=0.5$ to higher values of $x$.

It can not be ruled out at this stage that displacement of the sensors due to the change of sensor bias did take place in the experiment. Another possible explanation is that we have encountered a previously undetected problem in the alignment algorithm. 
We have been able to exclude the process of ballistic deficit as a possible explanation, mainly due to the lack of a clear thickness dependence in the observed shifts. Detailed simulations of the charge flow in the sensor have to be awaited before effects from varying contributions from charges at different depths can be kept responsible for an asymmetry for angled tracks. So far the standard simulations do not predict such large shifts for unirradiated sensors.

Further studies are being performed on how to better understand these shifts. We look forward to report more on this in the future.

\section{Acknowledgments}

The author wishes to thank Alison Bates, Jan Buytaert, Paula Collins, Doris Eckstein, Lars Eklund, Tomáš Laštovička and Chris Parkes for the many valuable discussions. Credit should also go to Juan Palacios and Steve Biagi for their early work in the study.

\section{References}

[1] LHCb Technical Design Report, Reoptimized Detector Design and Performance, CERN/LHCC, 2003-030.

[2] LHCb VELO Technical Design Report, CERN/LHCC, 2001-011.

[3] N. van Bakel et al, The Beetle Reference Manual, LHCb-2001-046.

[4] P. Bartalini et al, VELO telescope resolution and efficiency measurements, LCHb2000-099.

[5] The VeloTB user guide, http://www.cern.ch/jpalac/ doc/VeloTB/VeloTB_User_Guide.html

[6] The ROOT homepage, http://root.cern.ch.

[7] C. Parkes, Track Fit - Vertex Locator Test-Beam Software Description LCHb-2001038 VELO

[8] F. James, MINUIT Reference Manual, CERN Program Library entry D506 